\title{
Electric Field Facilitating Hole Transfer in Non-Fullerene Organic Solar Cells with a Negative HOMO Offset
}

\author{
Yanfeng Liu, ${ }^{\perp}$ Jianyun Zhang, ${ }^{\perp}$ Guanqing Zhou, Feng Liu, Xiaozhang Zhu, ${ }^{*}$ and Fengling Zhang*
}

Cite This: J. Phys. Chem. C 2020, 124, 15132-15139

Read Online

ABSTRACT: The record high photoinduced current and power conversion efficiencies of organic solar cells (OSCs) should be attributed to the significant contribution of non-fullerene electron acceptors via hole transfer to electron donors and/or a pronounced decrease in energy losses for exciton dissociation by aligned highest occupied molecular orbitals (HOMOs) or lowest unoccupied molecular orbitals (LUMOs). However, the hole transfer mechanism in those highly efficient non-fullerene OSCs with small HOMO offsets has not been extensively studied and fully understood, yet. Herein, we comparatively study the hole transfer kinetics in two OSCs with a positive $(0.05 \mathrm{eV})$ and a negative $(-0.07 \mathrm{eV}) \mathrm{HOMO}$ offset $(\Delta \mathrm{HOMO})$ based on polymer donor
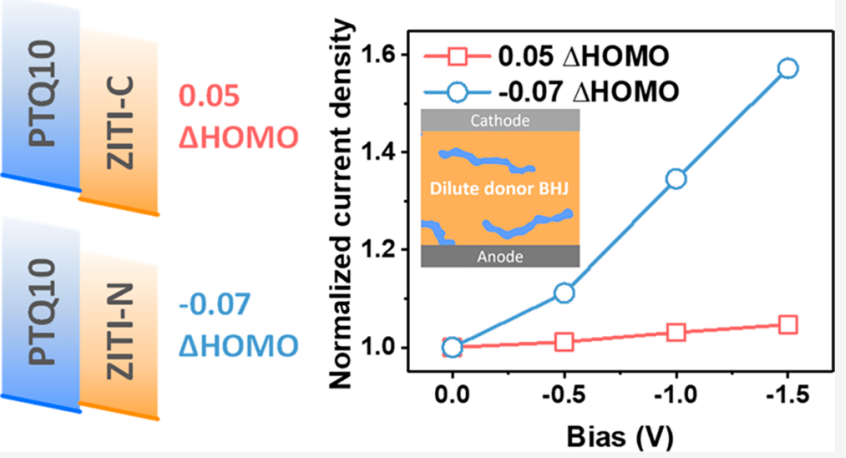
PTQ10 paired with non-fullerene acceptors ZITI-C or ZITI-N. Short-circuit current densities $\left(J_{\text {sc }}\right)$ of 20.42 and $12.81 \mathrm{~mA} \mathrm{~cm}{ }^{-2}$ are achieved in the OSCs based on PTQ10:ZITI-C $(\Delta H O M O=$ $0.05 \mathrm{eV})$ and PTQ10:ZITI-N $(\triangle \mathrm{HOMO}=-0.07 \mathrm{eV})$ with an optimized donor (D):acceptor $(\mathrm{A})$ ratio of 1:1, respectively, despite the small and even negative $\triangle \mathrm{HOMO}$. Results from time-resolved transient absorption spectroscopy show slower hole transfer (14.3 ps) in PTQ10:ZITI-N than that (3.7 ps) in PTQ10:ZITI-C. To understand the decent $J_{\text {sc }}$ value in the OSCs of PTQ10:ZITI-N, the temperature and electric field dependences of hole transfer are investigated in low-donor-content OSCs (D:A ratio of 1:9) in which photocurrent is dominated by the contribution via hole transfer from ZITI-N to PTQ10. Devices based on PTQ10:ZITI-C and PTQ10:ZITI-N show similar free charge generation behavior as a function of temperature, whereas the external quantum efficiencies of the PTQ10:ZITI-N device exhibit a much stronger bias dependence than that of PTQ10:ZITI-C, which suggests that the electric field facilitates exciton dissociation in PTQ10:ZITI-N where the energetic driving force alone cannot efficiently dissociate excitons.

\section{INTRODUCTION}

Efficient charge generation and transport processes have been systematically optimized in the field of organic solar cells (OSCs) via fine-tuning the morphologies and energy levels of donors and acceptors in active layers, along with the rational design of novel photovoltaic materials. ${ }^{1,2}$ The photocurrent generation in working OSCs can be decomposed into four steps, which are exciton formation upon photon absorption, diffusion of excitons in polymer or small molecule phases, exciton dissociation or free charge carrier generation at the donor:acceptor (D:A) interfaces, and finally charge transport (diffusion/drift) in bulk-heterojunctions (BHJs) toward and extraction at the corresponding electrodes.

For the last two steps, additional energetic driving forces are required to dissociate the excitons and drift holes/electrons to their corresponding electrodes, respectively. The driving force for exciton dissociation is mainly from the energy differences of the lowest unoccupied molecular orbitals (LUMOs) or the highest occupied molecular orbitals (HOMOs) between electron donors and acceptors. ${ }^{3-5}$ The forces to drift the charge carriers are mainly from the build-in potentials, which are created by the energic difference between the two extraction layers or electrodes. Early studies on fullerenebased OSCs concluded that a certain amount (around $0.3 \mathrm{eV}$ ) of LUMO or HOMO offsets ( $\triangle$ LUMO or $\triangle \mathrm{HOMO}$ ) is compulsory for efficient generation of photocurrent due to the formation of tight bonding excitons upon photon absorption in most organic semiconductors with relatively small dielectric constants. $^{6-8}$ As a result, the tightly bound electron-hole pairs can only be dissociated into free charge carriers by a larger energetic driving force than their mutual Coulomb attraction; otherwise, excitons will recombine right away.

Recent studies, however, based on efficient non-fullerene acceptor (NFA) OSCs, revealed that efficient charge generation could also be achieved in the NFA OSCs with reduced $\triangle \mathrm{LUMO}$ and $\triangle \mathrm{HOMO}$, as small as $0.08 \mathrm{eV}$ for

Received: June 22, 2020

Revised: June 24, 2020

Published: June 24, 2020 
(a)

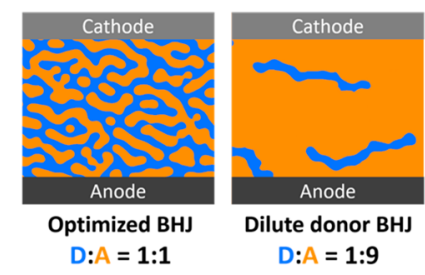

(c)

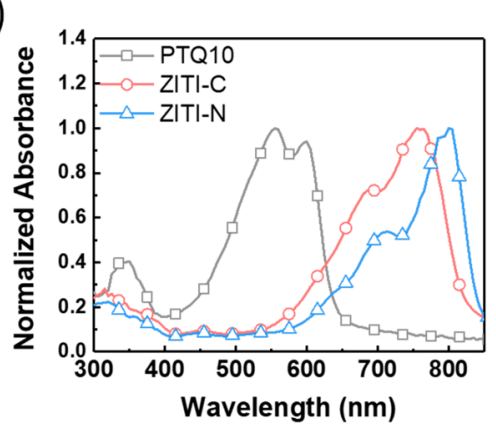

(b)

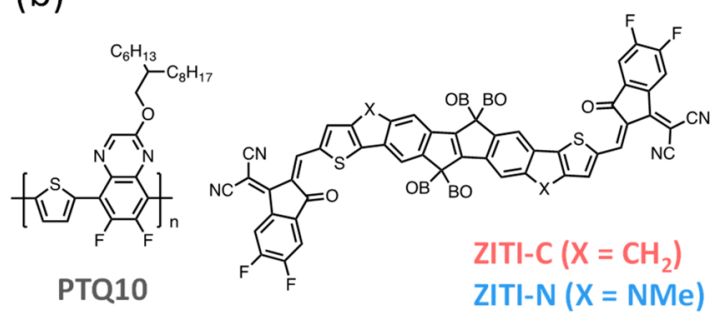

(d)

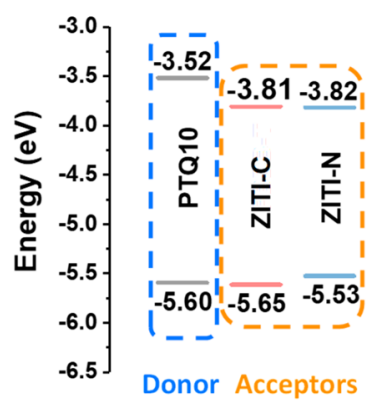

Figure 1. (a) Scheme of D and A distribution in optimized and dilute donor OSCs. (b) Molecular structures of PTQ10, ZITI-C, and ZITI-N. (c) Absorption spectra of the corresponding materials and (d) their energy levels.

$\triangle \mathrm{LUMO}$ and near $0 \mathrm{eV}$ for $\triangle \mathrm{HOMO}$, between electron donors and acceptors. ${ }^{9,10}$ In addition, solar cells under a negative $\triangle \mathrm{HOMO}$ of $-0.05 \mathrm{eV}$ based on PTQ10 and DFPCIC were also reported in a recent publication. ${ }^{11}$ Surprisingly, even with this undesired HOMO pair, the hole transfer from DF-PCIC to PTQ10 is not completely blocked by the low-lying HOMO of the donor, as indicated by the moderate short-circuit current densities $\left(J_{\mathrm{sc}}\right)$ of $6.51 \mathrm{~mA} \mathrm{~cm}{ }^{-2}$ and the fill factor (FF) of $54.13 \%$ in the corresponding devices. These observations are contrary to the previous common awareness that at least $0.3 \mathrm{eV}$ of $\triangle \mathrm{LUMO}$ or $\triangle \mathrm{HOMO}$ is necessary for efficient exciton dissociation.

Various hypotheses are proposed to interpret these phenomena. ${ }^{12-14}$ Studies revealed that differences in the degree of disorder between polymer domains and intermolecular interactions between the donor and acceptor can result in a hundreds of millielectronvolt shift in the HOMO levels of the polymers in $\mathrm{BHJ} .{ }^{15}$ These energy level shifts should be considered for the driving force calculation, and, more importantly, research highlighted that this energetic disorder could assist in the charge separation. ${ }^{16,17}$ In addition to the energetic disorder, charge carrier delocalization and thermal energy are reported to contribute to the charge separation process. ${ }^{13,18-21}$ For example, in 2018, Ifor Samuel and coworkers reported that the increase of hole polaron delocalization on polymer chains provided the driving force for charge separation by lowering the free energy of the spatially separated charge pairs. ${ }^{13}$ It is promising that Dieter Neher and co-workers showed that the majority of free charge generation occurs via thermalized charge transfer (CT) states, and for all blends in their study, activation energies for charge separation are on the same order of those at room temperature. The authors concluded that thermal energy at room temperature is sufficient to separate charge transfer states into free charges, which means the binding energy of the $\mathrm{CT}$ excitons in the blends in their study is much smaller than was commonly believed. $^{21}$ Furthermore, recent studies on free charge generation based on a high-efficient PM6:Y6 system by the same group provided a new perspective on charge separation in OSCs, which is that the Coulomb binding of the CT state can be overcome by the electrostatic potential that originated from the special molecular architecture of $\mathrm{Y} 6 .^{22}$

Generally, electrostatic fields that originated from the work function difference between the two electrodes are believed not to be sufficient to split the strongly bound excitons in the OSCs. ${ }^{7}$ The contribution of the electric fields to excitons dissociation has not been widely considered because the $\triangle$ LUMO or $\triangle \mathrm{HOMO}$ in many efficient $\mathrm{BHJ}$ systems could provide substantial energy for exciton dissociation where the donors and acceptors achieve an ideally cascade-type energy level alignment as proved by efficient photoluminescence (PL) quenching. ${ }^{23-25}$ However, the charge generation does exhibit a field-dependent feature, for instance, photocurrent affected by modulating the build-in potentials of the OSCs via employing different hole collecting electrodes. ${ }^{26}$ In 2018, Yana Vaynzof and co-workers demonstrated that the internal electric field facilitates the singlet exciton dissociation in the device based on PffBT4T-2OD:PC ${ }_{71} \mathrm{BM}^{27}$ It is time to re-examine the contribution of the external and internal electric field to charge generation in the NFA OSCs where the physics of photocurrent generation has been transformed into a new era by the development of efficient NFA-based systems with reduced energy offsets.

Recently, a systematic study on the correlation between the $\triangle \mathrm{HOMO}$ and device performance was conducted in ternary devices composed of the donor PTQ10 and two acceptors (ZITI-S and ZITI-N), where the $\triangle$ HOMO could be continuously modulated from 0.2 to $-0.07 \mathrm{eV}$ by varying the ratios of ZITI-S and ZITI-N from 1:0 to 0:1. ${ }^{5}$ The study concluded that a minimum $\triangle \mathrm{HOMO}$ (around $0.04 \mathrm{eV}$ ) is required to guarantee efficient exciton dissociation. However, the unexpected power conversion efficiency (PCE) of the 7.06\% PTQ10:ZITI-N device, along with the decent $J_{\text {sc }}$ of $12.03 \mathrm{~mA} \mathrm{~cm}^{-2}$, aroused our curiosity about the mechanism of hole transfer with a $\triangle \mathrm{HOMO}$ of $-0.07 \mathrm{eV}$. 
Herein, the hole transfer kinetics in PTQ10:ZITI-N devices is comparatively studied with reference solar cells based on the PTQ10 blend with ZITI-C, ${ }^{28}$ which gives a $\triangle \mathrm{HOMO}$ of 0.05 $\mathrm{eV}$, in two different stoichiometries (D:A ratios of $1: 1$ and 1:9). The devices of $1: 1$ based on PTQ10:ZITI-C exhibited a PCE of $13.1 \%$ with a $J_{\mathrm{sc}}$ of $20.4 \mathrm{~mA} \mathrm{~cm}^{-2}$, which outperformed its ZITI-N counterparts (PCE of 7.4\%). The devices of 1:9, also known as a dilute donor BHJ (Figure 1a), ${ }^{29-32}$ presented a PCE of $9.2 \%$ with a $J_{\text {sc }}$ of $15.0 \mathrm{~mA} \mathrm{~cm}^{-2}$ (PTQ10:ZITI-C), but $1.27 \%$ with a $J_{\text {sc }}$ of $2.75 \mathrm{~mA} \mathrm{~cm}^{-2}$ (PTQ10:ZITI-N). Both devices show similar free charge generation behavior as a function of temperature. However, the external quantum efficiencies (EQEs) of PTQ10:ZITI-N exhibit stronger electric field dependence than those of PTQ10:ZITI-C, which indicates that the electric field facilitates hole transfer in the device PTQ10:ZITI-N and thus highlights the important contribution of the electric field to charge generation via hole transfer in the OSCs with a negative $\triangle \mathrm{HOMO}$.

\section{EXPERIMENTAL SECTION}

Materials. ZITI-C and ZITI-N were prepared according to a previous publication. ${ }^{28}$ PTQ10 was purchased from Derthon Optoelectronic Materials Science Technology Co., Ltd. PDINO was purchased from 1-Material Inc. PEDOT:PSS (4083) was purchased from Heraeus. All materials were used as received.

Optical and Electrochemical Properties of Photoactive Materials. The absorption spectra of materials were tested by a Lambda 900 spectrometer from PerkinElmer. Cyclic voltammetry $(\mathrm{CV})$ measurements were performed with a CHI620D potentiostat. All measurements were carried out in a one-compartment cell under a nitrogen atmosphere, equipped with a glassy-carbon electrode, a platinum counter electrode, and an $\mathrm{Ag} / \mathrm{Ag}^{+}$reference electrode with a scan rate of $100 \mathrm{mV} \mathrm{s}^{-1}$. The supporting electrolyte was a $0.1 \mathrm{~mol} \mathrm{~L}^{-1}$ $\mathrm{CH}_{3} \mathrm{CN}$ solution of tetrabutylammonium perchlorate (TBAP). All potentials were corrected against $\mathrm{Fc} / \mathrm{Fc}^{+}$. $\mathrm{CV}$ was measured with a scan rate of $100 \mathrm{mV} \mathrm{s}^{-1}$.

Device Fabrication and Performance Measurements. OSCs were fabricated using ITO-coated glass substrates $(15 \Omega$ $\square^{-1}$ ), which were cleaned with deionized water, acetone, and isopropyl alcohol in successive 20 min sonication steps and by applying a final $20 \mathrm{~min}$ oxygen plasma treatment to eliminate any remaining organic component. A thin layer (ca. $30 \mathrm{~nm}$ ) of PEDOT:PSS was first spin-coated on the precleaned ITOcoated glass substrates at $3000 \mathrm{rpm}$ and baked at $150{ }^{\circ} \mathrm{C}$ for 15 min under ambient conditions. The substrates were then transferred into a nitrogen-filled glovebox. Subsequently, the active layers were spin-coated from chloroform solution with the same optimal D:A weight ratios of $1: 1$ and 1:9 for both PTQ10:ZITI-C (or PTQ10:ZITI-N) blends with a total concentration of $18 \mathrm{mg} \mathrm{mL}^{-1}$ and then treated with thermal annealing at $120^{\circ} \mathrm{C}$ for $10 \mathrm{~min}$. PDINO then was spin-coated on the active layer by $3000 \mathrm{rpm}$ from alcohol solution. At the final stage, the substrates were pumped down in high vacuum, and aluminum $(100 \mathrm{~nm})$ was thermally evaporated onto the active layer. Shadow masks were used to define the OSC active area $\left(0.047 \mathrm{~cm}^{2}\right)$ of the devices.

The current density-voltage $(J-V)$ characteristics of unencapsulated photovoltaic devices were measured under $\mathrm{N}_{2}$ using a Keithley 2400 source meter. A $300 \mathrm{~W}$ xenon arc solar simulator (Oriel) with an AM 1.5 global filter operated at $100 \mathrm{~mW} \mathrm{~cm} \mathrm{~cm}^{-2}$ was used to simulate the AM 1.5G solar irradiation. The illumination intensity was corrected by using a silicon photodiode with a protective KG5 filter calibrated by the National Renewable Energy Laboratory (NREL).

For the $J-V$ characteristics as a function of temperature, the device was placed in a sealed cycle helium cryostat with a compressor and oil pump, illuminated with an LSH-7320 AM $1.5 \mathrm{G}$ solar simulator, and the device performance was measured using a Keithley 2400 source meter. The EQE was performed using a QER3011 from Enli Technology Co., Ltd.

Atomic Force Microscopy (AFM) and Transmission Electron Microscopy (TEM). AFM images of the thin films were obtained on a NanoscopeIIIa AFM (Digital Instruments) operating platform in tapping mode. TEM observation was performed on a JEOL 2200FS at $160 \mathrm{kV}$ accelerating voltage.

Time-Resolved Transient Absorption (TA) Spectroscopy. For the femtosecond TA spectroscopy, samples were first pumped at $750 \mathrm{~nm}$ beam split from a $\mathrm{Yb}: \mathrm{KGW}$ laser and then probed with a white light continuum, which was converted by a YAG plate from the same laser source. The pump and probe overlapped on the sample at an angle of less than $10^{\circ}$. The transmitted probe light from the sample was collected by a linear charge-coupled device array.

$\mathrm{PL}$, Electroluminescence (EL), and Fourier Transform Photocurrent Spectroscopy (FTPS) Measurements. PL spectra were collected using an Andor spectrometer (Shamrock sr-303i-B, with a Newton EM-CCD detector) excited by a $532 \mathrm{~nm}$ laser source. EL spectra were detected by the same Newton EM-CCD detector and a Keithley 2400 sourcemeter. The data of EL quantum efficiency $\left(\mathrm{EQE}_{\mathrm{EL}}\right)$ were also obtained from a homemade detection system, which included a Hamamatsu silicon photodiode 1010B, a Keithley 2400 sourcemeter, and a Keithley 485 picoammeter. The FTPS-EQE spectra were measured by an in-house-built system based on Vertex 70 Fourier transform infrared spectroscopy (FTIR) from Bruker Optics.

\section{RESULTS AND DISCUSSION}

To understand the mechanism of hole transfer in the OSCs of PTQ10:ZITI-N, in this work, PTQ10:ZITI-C with a $\triangle H O M O$ of $0.05 \mathrm{eV}$ is selected as the reference sample. The molecular structures of the three materials used in this study are shown in Figure $1 \mathrm{~b}$ together with their absorption spectra and the energy levels (Figure 1c and d). The energy levels of these materials are measured by $\mathrm{CV}$ in thin films (Figure S1), and the $\mathrm{CV}$ results are consistent with those of previous publications. $^{5,28}$ Besides, the negative $\triangle$ HOMO between PTQ10 and ZITI-N is further confirmed by ultraviolet photoelectron spectroscopy measurements ${ }^{5}$ and the radiative efficiency measurements, which will be discussed hereafter.

Performance of the Optimized OSCs. First, the effect of $\Delta$ HOMOs on the performance of optimized OSCs (D:A ratio of $1: 1$ ) is studied with the conventional device geometry (ITO/PEDOT:PSS/active layer/PDINO/Al). The $J-V$ and EQE curves are presented in Figure 2. Photovoltaic performances are summarized in Table 1. As expected, the PTQ10:ZITI-C devices with a positive $0.05 \mathrm{eV} \Delta \mathrm{HOMO}$ show superior performance, with a $J_{\mathrm{sc}}$ of $24.42 \mathrm{~mA} \mathrm{~cm}^{-2}$, open circuit voltage $\left(V_{\mathrm{oc}}\right)$ of $0.93 \mathrm{~V}, \mathrm{FF}$ of $68.9 \%$, and PCE of $13.1 \%$. These values are comparable to those of the J71:ZITI-N devices that have the same $\triangle H O M O$ as in our previous report, ${ }^{28}$ which prove once again that the efficient charge transfer can be achieved with a $\triangle \mathrm{HOMO}$ as small as $0.05 \mathrm{eV}$. The ZITI-N-based devices are less efficient, but still maintain a 
(a)

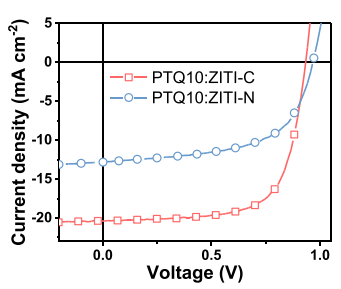

(b)

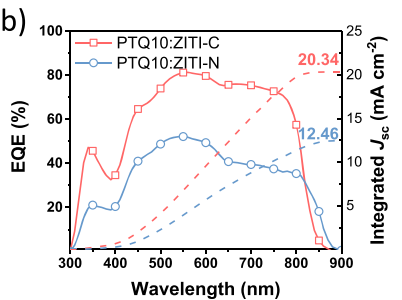

Figure 2. (a) $J-V$ and (b) EQE curves of BHJ devices based on ZITI$\mathrm{C}$ and ZITI-N.

decent $J_{\mathrm{sc}}$ of $12.81 \mathrm{~mA} \mathrm{~cm}{ }^{-2}, V_{\mathrm{oc}}$ of $0.97 \mathrm{~V}$, and $\mathrm{FF}$ of $59.7 \%$, as well as the PCE of $7.4 \%$ even with a $\triangle$ HOMO of $-0.07 \mathrm{eV}$. The higher $V_{\text {oc }}$ of ZITI-N-based devices is due to the decreased nonradiative energy losses, derived from both Shockley-Queisser $(\mathrm{S}-\mathrm{Q})$ limit theory calculations and the radiative efficiency measurements.

Similar morphologies of BHJ PTQ10:ZITI-N and PTQ10:ZITI-C are observed, as shown in Figure S2, by AFM and TEM, which exclude the impacts of nanostructures (domain size/interface) on the photovoltaic parameters, especially $J_{\mathrm{sc}}$. Accompanying a reduced $J_{\mathrm{sc}}$ as compared to the controlled device (PTQ10:ZITI-C), the EQE curve of the OSC based on PTQ10:ZITI-N also shows decreased value in all wavelength ranges, which indicates insufficient charge generation in the device that can be assigned to inefficient hole transfer from the acceptor to the donor due to the negative $\triangle$ HOMO. A slightly broader EQE profile of the PTQ10:ZITI$\mathrm{N}$ device than that of PTQ10:ZITI-C is consistent with their absorption spectra.

Charge Separation, Hole Transfer Kinetics, and Charge Recombination. To obtain an insight view of the charge separation kinetics, steady-state PL spectroscopy and time-resolved TA spectroscopy are conducted on PTQ10:ZITI-C and compared to those of PTQ10:ZITI-N blends. As shown in Figure $3 a$ and $b$, the PTQ10:ZITI-C blend shows completely quenched emission from ZITI-C, whereas an obvious emission from ZITI-N can be observed in the PTQ10:ZITI-N blend, which indicates less efficient charge separation, as well as the possible resonance energy transfer due to the mismatched energy level in the blends. Results from femtosecond TA spectroscopy further verified this conclusion. As presented in Figure $3 c$, there is a rise process of the PTQ10 ground-state bleaching in PTQ10:ZITI-C, which corresponds to the hole transfer process from ZITI-C in the blend film. The hole transfer time of the ZITI-C-based blend is $3.7 \mathrm{ps}$, which is shorter than the hole transfer time $(14.3 \mathrm{ps})$ in ZITI-N blended with PTQ10. All of the above results clearly demonstrate that a negative $\triangle \mathrm{HOMO}$ does hamper the efficiency of charge separation at the D:A interface, which results in less efficient solar cells. The complete TA spectra of PTQ10:ZITI-C and PTQ10:ZITI-N are shown in Figure S3.

The recombination dynamics of the devices are studied by measuring $J_{\mathrm{sc}}$ and $V_{\mathrm{oc}}$ under various light intensities. As shown in Figure S4, it is found that bimolecular recombination is dominated in both ZITI-C- and ZITI-N-based devices, and both devices share a similar recombination strength.

Energy Loss Analysis. The energy loss measurement is performed to investigate the influence of the different energetic offsets. First, FTPS and EL measurements are conducted for two blends and pristine ZITI-based devices. As shown in the FTPS and EL spectra in Figure S5, almost identical absorption and emission profiles in the low-energy range can be observed for the corresponding pristine and blend devices, which indicates that the energetic difference between the charge transfer states and the lowest excited states in the devices is negligible, common in recent device systems with minimum $\triangle$ HOMOs. $^{9}$ As presented in Figure S6, the $\mathrm{EQE}_{\mathrm{EL}}$ of the ZITI-N-based device is $3.1 \times 10^{-4}$, almost 1 order of magnitude higher than that of the ZITI-C-based device (1.1 $\left.\times 10^{-5}\right)$. The increased $\mathrm{EQE}_{\mathrm{EL}}$ in PTQ10:ZITI-N further confirmed the existence of a negative $\triangle \mathrm{HOMO}$, because during the $\mathrm{EQE}_{\mathrm{EL}}$ measurement, the device was operated in a LED mode, and the negative $\triangle \mathrm{HOMO}$ will become a favorable driving force for hole injection from $\mathrm{D}$ to $\mathrm{A} .^{33}$ The detailed calculations based on the $S-Q$ limit theory are presented in Table S1. The results show that the ZITI-N-based device has a lower nonradiative loss $(0.21 \mathrm{~V})$, identical to the calculation from $\mathrm{EQE}_{\mathrm{EL}}$, and the lower nonradiative loss should contribute to the higher $V_{\text {oc }}$ in the ZITI-N-based device.

Performance of Dilute Donor OSCs. For now, we have already demonstrated the influence of the negative $\triangle \mathrm{HOMO}$ on several critical processes of photocurrent generation, including charge separation and recombination, hole transfer, and the impact on voltage loss as well as the final device performance. However, a critical question remains unanswered, which is the origin of the driving force for hole transfer under the negative $\triangle$ HOMO. To take an in-depth view of the hole transfer in the working device, the concept "dilute donor BHJ", which only contains a small amount of polymer donor in the blend, is selected to construct OSCs. In dilute donor devices, due to the fact that the incident photons are mainly absorbed in the acceptor phase, the photocurrent is dominated by the contribution via the hole transfer process from acceptor to donor, whereas the contribution from electron transfer will be decreased accordingly. This feature makes the dilute donor concept a better model for studying the hole transfer process. ${ }^{34}$

Building upon the above advantages, the OSCs are fabricated on the basis of D:A blends in the ratio of 1:9 with the same conventional device geometry. The corresponding J$V$ and EQE curves, as well as the detailed photovoltaic performance, are shown in Figure 4 and Table 2. Surprisingly, the OSCs based on ZITI-C show an unexpected high efficiency around $9 \%$, with a decent $J_{\mathrm{sc}}$ of $15.00 \mathrm{~mA} \mathrm{~cm}{ }^{-2}$. Such a high

Table 1. Detailed Parameters of ZITI-C- and ZITI-N-Based BHJ Device Performance ${ }^{a}$

\begin{tabular}{|c|c|c|c|c|c|}
\hline active layer & $V_{\mathrm{oc}}(\mathrm{V})$ & $J_{\mathrm{sc}}\left(\mathrm{mA} \mathrm{cm} \mathrm{c}^{-2}\right)$ & $\mathrm{FF}(\%)$ & PCE (\%) & integrated $J_{\mathrm{sc}}\left(\mathrm{mA} \mathrm{cm}{ }^{-2}\right)$ \\
\hline PTQ10:ZITI-C & $\begin{array}{l}0.93 \\
(0.93 \pm 0.01)\end{array}$ & $\begin{array}{l}20.42 \\
(20.11 \pm 0.24)\end{array}$ & $\begin{array}{l}68.9 \\
(68.9 \pm 0.58)\end{array}$ & $\begin{array}{l}13.13 \\
(12.86 \pm 0.15)\end{array}$ & 20.34 \\
\hline PTQ10:ZITI-N & $\begin{array}{l}0.97 \\
(0.97 \pm 0.01)\end{array}$ & $\begin{array}{l}12.81 \\
(11.88 \pm 0.77)\end{array}$ & $\begin{array}{l}59.7 \\
(57.7 \pm 1.17)\end{array}$ & $\begin{array}{l}7.39 \\
(6.70 \pm 0.49)\end{array}$ & 12.46 \\
\hline
\end{tabular}

${ }^{a_{\text {The }}}$ average values with standard deviation are obtained from 20 devices. 
(a)

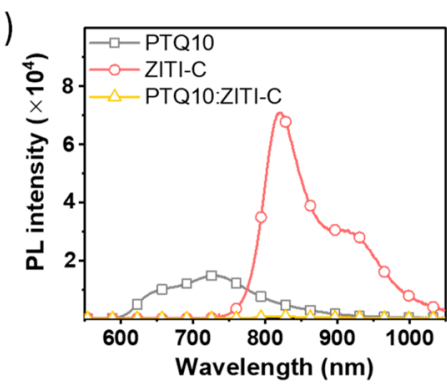

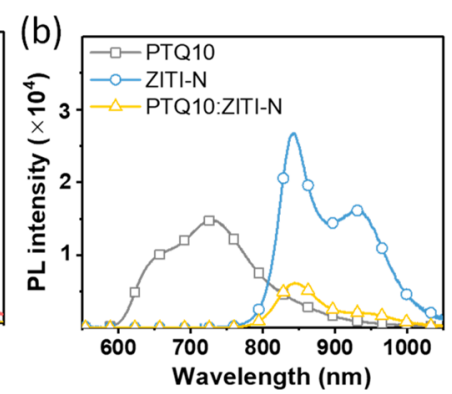

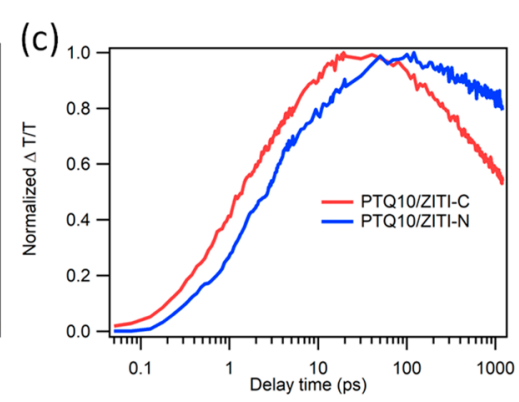

Figure 3. (a,b) Steady-state PL spectra of pristine PTQ10, ZITI-C, ZITI-N, and their blend films. Films are excited under a $532 \mathrm{~nm}$ laser. (c) TA kinetics of the hole transfer process of two blend films, where the TA data of PTQ10:ZITI-N are from a previous publication. ${ }^{5}$

(a)

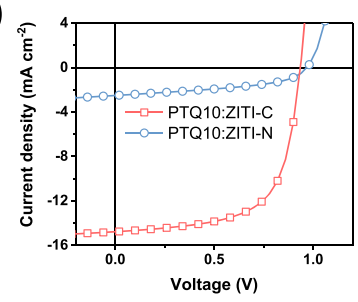

(b)

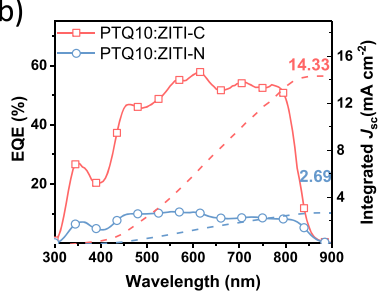

Figure 4. (a) $J-V$ and (b) EQE curves of dilute BHJ devices based on PTQ10:ZITI-C and PTQ10:ZITI-N.

performance demonstrates that the hole transfer process is quite efficient in the D:A interface. In this dilute donor BHJ, the main contribution of photocurrent is from the photon absorbed in the ZITI-C phase, as shown in Figure S7, and the following hole transfer from ZITI-C to PTQ10. In addition, the dilute donor devices based on ZITI-N also achieve a PCE of $1.3 \%$, with a $J_{s c}$ of $2.75 \mathrm{~mA} \mathrm{~cm}{ }^{-2}$ despite the negative $\triangle$ HOMO. It is worth noting that in the optimized configuration, the existence of hole transfer barrier halves the $J_{\text {sc }}$ of the ZITI-N-based device as compared to its ZITI-C counterpart, whereas in the dilute donor configuration, the $J_{\mathrm{sc}}$ of the ZITI-N-based device is less than one-fifth of the $J_{\mathrm{sc}}$ in the device based on ZITI-C. The much-reduced $J_{\mathrm{sc}}$ of PTQ10:ZITI-N indicates that the impact of the hole transfer process on photocurrent can be magnified in the dilute donor devices.

The FFs of OSCs are good indicators for transportation property in the devices. The FF of $65.5 \%$ in ZITI-C indicates balanced electron and hole transportation and efficient collection in both electrodes, which means that ZITI-C is an ambipolar conductor for both electrons and holes. On the other hand, the ZITI-N devices show a small FF of $47.3 \%$, which could be due to the decreased electron/hole mobilities of the pure ZITI-N material, as indicated in Figure S8 and Table S2. We should point out that the differences in mobilities between ZITI-C and ZITI-N should not be the main reason for smaller $J_{\mathrm{sc}}$ and FF in ZITI-N devices because

ZITI-C and ZITI-N have similar behavior when they combine with $\mathrm{J} 71$ as demonstrated in a previous report. ${ }^{28}$

Temperature-Dependent Charge Generation. To probe the driving force for hole transfers in the OSCs with a $\Delta \mathrm{HOMO}=-0.07 \mathrm{eV}$, the charge generation behavior of diluted devices is first studied under different temperatures $(T)$, by continuously examining their $V_{\text {oc }}$ from room temperature $(300 \mathrm{~K})$ to $125 \mathrm{~K}$. Note that for PTQ10:ZITI$\mathrm{C}$, the $T$ range is from 300 to $200 \mathrm{~K}$, because the device broke down when $T$ was lower than $200 \mathrm{~K}$. In Figure 5a, a linear
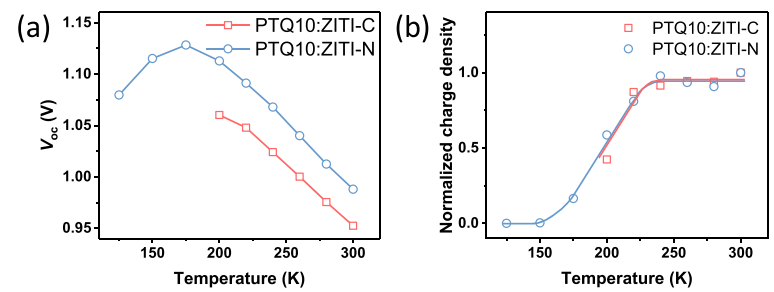

Figure 5. (a) Temperature-dependent $V_{\text {oc }}\left(V_{o c}-T\right)$ plot of PTQ10:ZITI-C and PTQ10:ZITI-N dilute devices. (b) Calculated temperature-dependent charge densities of the corresponding devices. The solid lines are guides for the eye.

relation in the $V_{\text {oc }}-T$ plot can be observed for both devices from 300 to $240 \mathrm{~K}$, then they start to deviate at lower $T$. Previous studies pointed out that this sublinear phenomenon in $V_{\text {oc }}-T$ plots is due to temperature-dependent charge separation, and the linear relation indicates charge separation is temperature independent in a certain temperature range. ${ }^{35,36}$ To further confirm this statement, the charge carrier densities in solar cells under the same temperature range are calculated following the method in the literature, ${ }^{37}$ and the results are shown in Figure 5b. For both dilute devices, the charge densities remain constant from room temperature to around $240 \mathrm{~K}$, which means that in both devices charge separation is not affected by temperature in a wide range. Therefore, in our case, we conclude that thermal energy similarly impacts the

Table 2. Photovoltaic Performance of Dilute BHJ Solar Cells Based on PTQ10:ZITI-C and PTQ10:ZITI-N ${ }^{a}$

$\begin{array}{cccccc}\text { active layer } & V_{\mathrm{oc}}(\mathrm{V}) & J_{\mathrm{sc}}\left(\mathrm{mA} \mathrm{cm}^{-2}\right) & \mathrm{FF}(\%) & \text { PCE }(\%) & \text { integrated } J_{\mathrm{sc}}\left(\mathrm{mA} \mathrm{cm} \mathrm{cm}^{-2}\right) \\ \text { PTQ10:ZITI-C 1:9 } & 0.94 & 15.00 & 65.5 & 9.22 & 14.33 \\ & (0.93 \pm 0.01) & (15.50 \pm 0.65) & (60.6 \pm 4.88) & (8.76 \pm 0.50) & \\ \text { PTQ10:ZITI-N 1:9 } & 0.98 & 2.75 & 47.3 & 1.27 & 2.69 \\ & (0.97 \pm 0.01) & (2.36 \pm 0.21) & (46.0 \pm 1.51) & (1.08 \pm 0.10) & \end{array}$

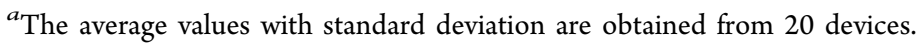



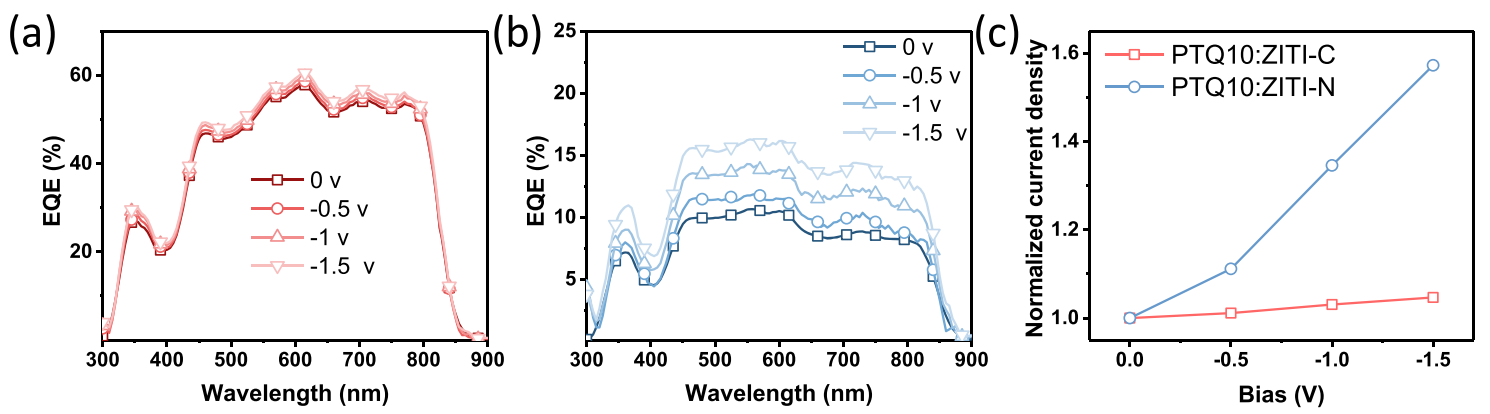

Figure 6. EQE curves of (a) PTQ10:ZITI-C and (b) PTQ10:ZITI-N dilute devices under different reverse bias. (c) Normalized $J_{\text {sc }}$ values in dilute donor devices under different biases.

charge separation behavior in PTQ10:ZITI-C and PTQ10:ZITI-N dilute devices.

Electric Field-Dependent Charge Generation. Next, the field-dependent EQEs of devices based on the 1:9 ratio are measured and presented in Figure 6a and b. EQE spectra of both devices go up when increasing the reversed bias, which indicates acceleration of the charge generation processes by the bias. However, the degree of variation trends is very different in the cases of ZITI-C and ZITI-N under the same bias gradience. As summarized in Table 3, from 0 to $-0.5 \mathrm{~V}$, the

Table 3. Integrated $J_{s c}$ Values from Dilute Devices and the Corresponding Enhancement Percentage as Compared to the $J_{\mathrm{sc}}$ Value under $0 \mathrm{~V}$

\begin{tabular}{cccccc} 
& \multicolumn{2}{c}{ PTQ10:ZITI-C } & & \multicolumn{2}{c}{ PTQ10:ZITI-N } \\
\cline { 2 - 3 } \cline { 5 - 6 } $\begin{array}{c}\text { applied } \\
\text { bias }(\mathrm{V})\end{array}$ & $\begin{array}{c}\text { integrated } J_{\text {sc }} \\
\left(\mathrm{mA} \mathrm{cm}^{-2}\right)\end{array}$ & $\begin{array}{c}\text { enhancement } \\
(\%)\end{array}$ & & $\begin{array}{c}\text { integrated } J_{\text {sc }} \\
\left(\mathrm{mA} \mathrm{cm}^{-2}\right)\end{array}$ & $\begin{array}{c}\text { enhancement } \\
(\%)\end{array}$ \\
0 & 14.33 & & & 2.69 & \\
-0.5 & 14.49 & 1.1 & & 2.99 & 11.2 \\
-1 & 14.77 & 3.1 & & 3.62 & 34.6 \\
-1.5 & 15.00 & 4.7 & & 4.23 & 57.2 \\
\hline
\end{tabular}

integrated $J_{s c}$ of the ZITI-C-based device shows a $1.1 \%$ enhancement, whereas the enhancement in the ZITI-N-based device is $11.2 \%$. Even slightly larger enhancements $(34.6 \%$ and $57.2 \%)$ are observed under larger biases $(-1.0$ and $-1.5 \mathrm{~V})$, as indicated in Figure $6 \mathrm{c}$ and Table 3, which are about 10 times those in the ZITI-C-based device under the same biases. The field-dependent EQE shows that a large bias generates a much stronger impact on the device with a negative $\triangle \mathrm{HOMO}$, and this bias-sensitive charge generation behavior illustrates that the electric field assists hole transfer, when the energetic driving force for hole transfer is lacking inside the blends.

\section{CONCLUSIONS}

In summary, the hole transfer kinetics is comparatively studied in the OSCs based on PTQ10:ZITI-C or ZITI-N with a $\triangle \mathrm{HOMO}$ of 0.05 or $-0.07 \mathrm{eV}$ in the stoichiometries of $1: 1$ and 1:9, respectively. The OSCs (1:1) based on PTQ10:ZITI$\mathrm{C}$ ensure an efficient hole transfer process, confirmed by a complete PL quenching, ultrafast hole transfer revealed by TA spectroscopy, as well as the superior device performance (PCE of $13.1 \%$ with $J_{\mathrm{sc}}$ of $20.42 \mathrm{~mA} \mathrm{~cm}^{-2}$ ). A smaller PCE of $7.4 \%$ and $J_{\mathrm{sc}}$ of $12.03 \mathrm{~mA} \mathrm{~cm}{ }^{-2}$ in OSCs of PTQ10:ZITI-N than those of PTQ10:ZITI-C indicate the significant impacts of $\triangle$ HOMO. Studies regarding hole transfer in dilute donor OSCs (1:9) as a function of temperature and electric field suggest similar free charge generation under different temperatures, but a much stronger bias dependent on the photocurrent in the PTQ10:ZITI-N device than that of PTQ10:ZITI-C, which highlights the contribution of the electric field on dissociate excitons via hole transfer in the OSCs with negative $\triangle$ HOMO. Therefore, enlarging the internal electric field of OSCs, for example, by selecting appropriate electrodes or/and interfacial layers, will be a feasible strategy to further increase the efficiency of non-fullerene OSCs.

\section{ASSOCIATED CONTENT}

\section{Supporting Information}

The Supporting Information is available free of charge at https://pubs.acs.org/doi/10.1021/acs.jpcc.0c05654.

$\mathrm{CV}$ results of active layer materials; absorption spectra of blend films with different D:A ratios; electron and hole mobilities of pristine ZITI-C and ZITI-N; AFM and TEM images and complementary TA spectra of the blend films; results of measured $J_{\mathrm{sc}}$ and $V_{\mathrm{oc}}$ values of the device with different active layers; and details of the energy loss calculation for the solar cells (PDF)

\section{AUTHOR INFORMATION}

\section{Corresponding Authors}

Xiaozhang Zhu - Beijing National Laboratory for Molecular Sciences, CAS Key Laboratory of Organic Solids, Institute of Chemistry, Chinese Academy of Sciences, Beijing 100190, China; School of Chemical Sciences, University of Chinese Academy of Sciences, Beijing 100049, China; O orcid.org/ 0000-0002-6812-0856; Email: xzzhu@iccas.ac.cn

Fengling Zhang - Department of Physics, Chemistry and Biology, Linköping University, Linköping SE-581 83, Sweden; ๑ orcid.org/0000-0002-1717-6307; Email: fengling.zhang@ liu.se

\section{Authors}

Yanfeng Liu - Department of Physics, Chemistry and Biology, Linköping University, Linköping SE-581 83, Sweden

Jianyun Zhang - Beijing National Laboratory for Molecular Sciences, CAS Key Laboratory of Organic Solids, Institute of Chemistry, Chinese Academy of Sciences, Beijing 100190, China; School of Chemical Sciences, University of Chinese Academy of Sciences, Beijing 100049, China

Guanqing Zhou - School of Chemistry and Chemical Engineering, Center for Advanced Electronic Materials and Devices, Shanghai Jiao Tong University, Shanghai 200240, China 
Feng Liu - School of Chemistry and Chemical Engineering, Center for Advanced Electronic Materials and Devices, Shanghai Jiao Tong University, Shanghai 200240, China

Complete contact information is available at:

https://pubs.acs.org/10.1021/acs.jpcc.0c05654

\section{Author Contributions}

${ }^{\perp}$ Y.L. and J.Z. contributed equally to this work.

\section{Notes}

The authors declare no competing financial interest.

\section{ACKNOWLEDGMENTS}

Y.L. and F.Z. acknowledge financial support from the Swedish Government Strategic Research Area in Material Science on Functional Materials at Linköping University (Faculty Grant SFO-Mat-LiU no. 200900971), the Swedish Research Council (2017-04123), the Knut and Alice Wallenberg Foundation (2016.0059) for Fellowship grand funding, and the China Scholarship Council (CSC). X.Z. thanks the National Key R\&D Program of China (2019YFA0705900, 2017YFA0204701) and the National Natural Science Foundation of China (21572234, 21661132006, 91833304) for their financial support.

\section{REFERENCES}

(1) Yuan, J.; Zhang, Y. Q.; Zhou, L. Y.; Zhang, G. C.; Yip, H. L.; Lau, T. K.; Lu, X. H.; Zhu, C.; Peng, H. J.; Johnson, P. A.; et al. Single-Junction Organic Solar Cell with Over 15\% Efficiency Using Fused-Ring Acceptor with Electron-Deficient Core. Joule 2019, 3, $1140-1151$.

(2) Zhou, Z.; Liu, W.; Zhou, G.; Zhang, M.; Qian, D.; Zhang, J.; Chen, S.; Xu, S.; Yang, C.; Gao, F.; et al. Subtle Molecular Tailoring Induces Significant Morphology Optimization Enabling over 16\% Efficiency Organic Solar Cells with Efficient Charge Generation. Adv. Mater. 2020, 32, 1906324.

(3) Chen, S.; Wang, Y.; Zhang, L.; Zhao, J.; Chen, Y.; Zhu, D.; Yao, H.; Zhang, G.; Ma, W.; Friend, R. H.; et al. Efficient Nonfullerene Organic Solar Cells with Small Driving Forces for Both Hole and Electron Transfer. Adv. Mater. 2018, 30, 1804215.

(4) Ren, G.; Schlenker, C. W.; Ahmed, E.; Subramaniyan, S.; Olthof, S.; Kahn, A.; Ginger, D. S.; Jenekhe, S. A. Photoinduced Hole Transfer Becomes Suppressed with Diminished Driving Force in Polymer-Fullerene Solar Cells While Electron Transfer Remains Active. Adv. Funct. Mater. 2013, 23, 1238-1249.

(5) Zhang, J.; Liu, W.; Zhou, G.; Yi, Y.; Xu, S.; Liu, F.; Zhu, H.; Zhu, $X$. Accurate Determination of the Minimum HOMO Offset for Efficient Charge Generation using Organic Semiconducting Alloys. Adv. Energy Mater. 2020, 10, 1903298.

(6) Clarke, T. M.; Durrant, J. R. Charge Photogeneration in Organic Solar Cells. Chem. Rev. 2010, 110, 6736-6767.

(7) Blom, P. W. M.; Mihailetchi, V. D.; Koster, L. J. A.; Markov, D. E. Device Physics of Polymer:Fullerene Bulk Heterojunction Solar Cells. Adv. Mater. 2007, 19, 1551-1566.

(8) Dennler, G.; Scharber, M. C.; Brabec, C. J. Polymer-Fullerene Bulk-Heterojunction Solar Cells. Adv. Mater. 2009, 21, 1323-1338.

(9) Qian, D.; Zheng, Z.; Yao, H.; Tress, W.; Hopper, T. R.; Chen, S.; Li, S.; Liu, J.; Chen, S.; Zhang, J.; et al. Design Rules for Minimizing Voltage Losses in High-Efficiency Organic Solar Cells. Nat. Mater. 2018, 17, 703-709.

(10) Liu, J.; Chen, S.; Qian, D.; Gautam, B.; Yang, G.; Zhao, J.; Bergqvist, J.; Zhang, F.; Ma, W.; Ade, H.; et al. Fast Charge Separation in a Non-Fullerene Organic Solar Cell with a Small Driving Force. Nat. Energy 2016, 1, 16089.

(11) Li, S.; Zhan, L.; Sun, C.; Zhu, H.; Zhou, G.; Yang, W.; Shi, M.; Li, C. Z.; Hou, J.; Li, Y.; et al. Highly Efficient Fullerene-Free Organic
Solar Cells Operate at Near Zero Highest Occupied Molecular Orbital Offsets. J. Am. Chem. Soc. 2019, 141, 3073-3082.

(12) Nakano, K.; Chen, Y.; Xiao, B.; Han, W.; Huang, J.; Yoshida, H.; Zhou, E.; Tajima, K. Anatomy of the Energetic Driving Force for Charge Generation in Organic Solar Cells. Nat. Commun. 2019, 10, 2520.

(13) Matheson, A. B.; Ruseckas, A.; Pearson, S. J.; Samuel, I. D. W. Hole Delocalization as a Driving Force for Charge Pair Dissociation in Organic Photovoltaics. Mater. Horiz. 2019, 6, 1050-1056.

(14) Wang, J.; Xu, J.; Yao, N.; Zhang, D.; Zheng, Z.; Xie, S.; Zhang, X.; Zhang, F.; Zhou, H.; Zhang, C.; et al. A Comparative Study on Hole Transfer Inversely Correlated with Driving Force in Two NonFullerene Organic Solar Cells. J. Phys. Chem. Lett. 2019, 10, 41104116.

(15) Sweetnam, S.; Graham, K. R.; Ngongang Ndjawa, G. O.; Heumuller, T.; Bartelt, J. A.; Burke, T. M.; Li, W.; You, W.; Amassian, A.; McGehee, M. D. Characterization of the Polymer Energy Landscape in Polymer:Fullerene Bulk Heterojunctions with Pure and Mixed Phases. J. Am. Chem. Soc. 2014, 136, 14078-14088.

(16) Athanasopoulos, S.; Schauer, F.; Nádaždy, V.; Weiß, M.; Kahle, F.-J.; Scherf, U.; Bässler, H.; Köhler, A. What is the Binding Energy of a Charge Transfer State in an Organic Solar Cell? Adv. Energy Mater. 2019, 9, 1900814.

(17) Hood, S. N.; Kassal, I. Entropy and Disorder Enable Charge Separation in Organic Solar Cells. J. Phys. Chem. Lett. 2016, 7, 44954500.

(18) Jakowetz, A. C.; Bohm, M. L.; Zhang, J.; Sadhanala, A.; Huettner, S.; Bakulin, A. A.; Rao, A.; Friend, R. H. What Controls the Rate of Ultrafast Charge Transfer and Charge Separation Efficiency in Organic Photovoltaic Blends. J. Am. Chem. Soc. 2016, 138, 1167211679.

(19) Shoaee, S.; Subramaniyan, S.; Xin, H.; Keiderling, C.; Tuladhar, P. S.; Jamieson, F.; Jenekhe, S. A.; Durrant, J. R. Charge Photogeneration for a Series of Thiazolo-Thiazole Donor Polymers Blended with the Fullerene Electron Acceptors PCBM and ICBA. Adv. Funct. Mater. 2013, 23, 3286-3298.

(20) Athanasopoulos, S.; Bassler, H.; Kohler, A. Disorder vs Delocalization: Which is More Advantageous for High-Efficiency Organic Solar Cells? J. Phys. Chem. Lett. 2019, 10, 7107-7112.

(21) Kurpiers, J.; Ferron, T.; Roland, S.; Jakoby, M.; Thiede, T.; Jaiser, F.; Albrecht, S.; Janietz, S.; Collins, B. A.; Howard, I. A.; et al. Probing the Pathways of Free Charge Generation in Organic Bulk Heterojunction Solar Cells. Nat. Commun. 2018, 9, 2038.

(22) Perdigon-Toro, L.; Zhang, H.; Markina, A.; Yuan, J.; Hosseini, S. M.; Wolff, C. M.; Zuo, G.; Stolterfoht, M.; Zou, Y.; Gao, F.; et al. Barrierless Free Charge Generation in the High-Performance PM6:Y6 Bulk Heterojunction Non-Fullerene Solar Cell. Adv. Mater. 2020, 32, 1906763.

(23) Vandewal, K.; Ma, Z.; Bergqvist, J.; Tang, Z.; Wang, E.; Henriksson, P.; Tvingstedt, K.; Andersson, M. R.; Zhang, F.; Inganäs, O. Quantification of Quantum Efficiency and Energy Losses in Low Bandgap Polymer:Fullerene Solar Cells with High Open-Circuit Voltage. Adv. Funct. Mater. 2012, 22, 3480-3490.

(24) Gélinas, S.; Rao, A.; Kumar, A.; Smith, S. L.; Chin, A. W.; Clark, J.; van der Poll, T. S.; Bazan, G. C.; Friend, R. H. Ultrafast Long-Range Charge Separation in Organic Semiconductor Photovoltaic Diodes. Science 2014, 343, 512-516.

(25) Bakulin, A. A.; Rao, A.; Pavelyev, V. G.; van Loosdrecht, P. H.; Pshenichnikov, M. S.; Niedzialek, D.; Cornil, J.; Beljonne, D.; Friend, R. H. The Role of Driving Energy and Delocalized States for Charge Separation in Organic Semiconductors. Science 2012, 335, 13401344.

(26) Zhang, F.; Johansson, M.; Andersson, M. R.; Hummelen, J. C.; Inganäs, O. Polymer Photovoltaic Cells with Conducting Polymer Anodes. Adv. Mater. 2002, 14, 662-665.

(27) Weu, A.; Hopper, T. R.; Lami, V.; Kreß, J. A.; Bakulin, A. A.; Vaynzof, Y. Field-Assisted Exciton Dissociation in Highly Efficient PffBT4T-2OD:Fullerene Organic Solar Cells. Chem. Mater. 2018, 30, $2660-2667$. 
(28) Zhang, J.; Liu, W.; Zhang, M.; Liu, Y.; Zhou, G.; Xu, S.; Zhang, F.; Zhu, H.; Liu, F.; Zhu, X. Revealing the Critical Role of the HOMO Alignment on Maximizing Current Extraction and Suppressing Energy Loss in Organic Solar Cells. iScience 2019, 19, 883-893.

(29) Xu, L.; Wang, J.; Villa, M. d. A.; Daunis, T. B.; Lee, Y.-J.; Malko, A. V.; Hsu, J. W. P. Quantitative Analyses of Competing Photocurrent Generation Mechanisms in Fullerene-Based Organic Photovoltaics. J. Phys. Chem. C 2016, 120, 16470-16477.

(30) Collado-Fregoso, E.; Pugliese, S. N.; Wojcik, M.; Benduhn, J.; Bar-Or, E.; Perdigon Toro, L.; Hormann, U.; Spoltore, D.; Vandewal, K.; Hodgkiss, J. M.; et al. Energy-Gap Law for Photocurrent Generation in Fullerene-Based Organic Solar Cells: The Case of Low-Donor-Content Blends. J. Am. Chem. Soc. 2019, 141, 23292341.

(31) Hussain, K.; Kaiser, W.; Gagliardi, A. Effect of Polymer Morphology on Dilute Donor Organic Solar Cells. J. Phys. Chem. C 2020, 124, 3517-3528.

(32) Albes, T.; Xu, L.; Wang, J.; Hsu, J. W. P.; Gagliardi, A. Origin of Photocurrent in Fullerene-Based Solar Cells. J. Phys. Chem. C 2018, 122, 15140-15148.

(33) Xie, Y.; Wang, W.; Huang, W.; Lin, F.; Li, T.; Liu, S.; Zhan, X.; Liang, Y.; Gao, C.; Wu, H.; et al. Assessing the Energy Offset at the Electron Donor/Acceptor Interface in Organic Solar Cells Through Radiative Efficiency Measurements. Energy Environ. Sci. 2019, 12, $3556-3566$

(34) Song, Y.; Schubert, A.; Liu, X.; Bhandari, S.; Forrest, S. R.; Dunietz, B. D.; Geva, E.; Ogilvie, J. P. Efficient Charge Generation via Hole Transfer in Dilute Organic Donor-Fullerene Blends. J. Phys. Chem. Lett. 2020, 11, 2203-2210.

(35) Vandewal, K.; Tvingstedt, K.; Gadisa, A.; Inganäs, O.; Manca, J. V. Relating the Open-Circuit Voltage to Interface Molecular Properties of Donor:Acceptor Bulk Heterojunction Solar Cells. Phys. Rev. B: Condens. Matter Mater. Phys. 2010, 81, 125204.

(36) Rauh, D.; Wagenpfahl, A.; Deibel, C.; Dyakonov, V. Relation of Open Circuit Voltage to Charge Carrier Density in Organic Bulk Heterojunction Solar Cells. Appl. Phys. Lett. 2011, 98, 133301.

(37) Gao, F.; Tress, W.; Wang, J.; Inganas, O. Temperature Dependence of Charge Carrier Generation in Organic Photovoltaics. Phys. Rev. Lett. 2015, 114, 128701. 